\title{
Brownian Motion at the Speed of Light: A New Lorentz Invariant Family of Processes
}

\author{
Maurizio Serva ${ }^{1}$ (i) \\ Received: 6 October 2020 / Accepted: 17 February 2021 / Published online: 11 March 2021 \\ (c) The Author(s) 2021
}

\begin{abstract}
We consider here a new family of processes which describe particles which only can move at the speed of light $c$ in the ordinary 3D physical space. The velocity, which randomly changes direction, can be represented as a point on the surface of a sphere of radius $c$ and its trajectories only may connect points of this variety. A process can be constructed both by considering jumps from one point to another (velocity changes discontinuously) and by continuous velocity trajectories on the surface. We recently proposed to follow this second strategy assuming that the velocity is described by a Wiener process (which is isotropic only in the 'rest frame') on the surface of the sphere. Using both Ito calculus and Lorentz boost rules, we succeed here in characterizing the entire Lorentz-invariant family of processes. Moreover, we highlight and describe the short-term ballistic behavior versus the long-term diffusive behavior of the particles in the $3 \mathrm{D}$ physical space.
\end{abstract}

Keywords Brownian motion · Wiener process · Relativity · Lorentz boost · Ito calculus

\section{Introduction}

Brownian motion is a physical phenomenon which was historically modeled by the Wiener process, both directly identifying the particle trajectories with the realizations of the process and indirectly assuming that the particle velocity evolves according to a Ornstein-Uhlenbeck process. Although in the last century the Wiener process has been used to describe a variety of phenomena in finance, biology, engineering, electronics and so on, its name remains strictly tied to the description of the motion of random particles.

If one tries to extend its use to the description of the random motion of relativistic particles, one clashes against one of its more characteristic properties: trajectories are not differentiable, which means infinite speed while relativity only allows luminal or subluminal velocities. This fact doesn't imply that it is useless, on the contrary the relativistic Brownian motion can be

Communicated by Michael Kiessling.

Maurizio Serva

serva@univaq.it

1 Dipartimento di Ingegneria e Scienze dell'Informazione e Matematica, Università dell'Aquila, L'Aquila, Italy 
still modeled via a variety of modified Langevin equations which produce trajectories with a speed which is never superluminal. We just quote [1-21] which are a few of the studies which followed this strategy in the last fifty years.

In a recent research [22] we considered the extreme case in which a particle moves at the speed of light, the aim was to produce a probabilistic tool which is related to the Brownian motion of light-speed particles in the same way as the Wiener process is related to the Brownian motion of classical particles. We do not derive here the erratic motion of a light-speed particle by some limit procedure which involves collisions with other particles or obstacles as in [23], but we directly provide the mathematical framework. Although it appears very difficult to imagine a physical device where a mass-less particle is trapped and scattered as a photon in a box of mirrors, this is, indeed, closer to reality of what one could think. Almost one century ago, Albert Einstein theoretically conceived a box in which a single photon could be trapped in order to measure the relationship between mass and energy. Recently a team of physicists have created this box, a device that snares a photon up to half a second [24].

One more reason for searching a Wiener description of relativistic random particles is the possibility to extend the analogy between the Feynman integral and the Wiener integral (Feynman-Kac formula) to the relativistic quantum domain. The Schrödinger equation is solved by the Feynman integral while the heat equation, which is connected to the first by analytic continuation, is solved by the Wiener integral. The relativistic versions of the Schrödinger equation are the Klein-Gordon (zero spin particles) and Dirac (spin one half) equations. Both are hyperbolic equations (Dirac equation in its second order formulation). Analytic continuation gives rise to elliptic equations, the point is: which process is associated to the elliptic equations?

The first answers were given in [25-29] and later implemented in [30,31] where the Wiener process was still the main ingredient, but a four dimensional one with both position and time following trajectories which are the realizations of a Wiener process with the proper time as index. The proper time is then eliminated by a procedure based on hitting times. Nevertheless, the resulting process is unphysical since the speed is not bounded and the whole construction only results in a tool for obtaining a probabilistic solution of some elliptic equations. If one forces the approach to the realm of physics one has to abandon Markov property and the single particle picture [32].

There is a third way to approach the relativistic problem with a process which is physical and allows to construct the solution of the quantum hyperbolic equations. In 1956 the Polish physicist and mathematician Mark Kac considered a (1+1)-dimensional process (one space dimension + time) where the particle travels at speed of light (left or right) and randomly inverts its velocity and he proved that the associated probability density satisfies the telegrapher equation [33].

About thirty years after the Kac pioneering work, Gaveau et al. noticed that the telegrapher equation could be easily associated both to the Dirac equation in $1+1$ dimensions (first order formulation) and to the Klein-Gordon equation also in 1+1 dimensions (second order formulation). Using this equivalence they were able to give a probabilistic solution (by the backward Kolmogorov equation) to these fundamental quantum equations [34]. This result was later refined and extended in [35,36]. The weak point was that both Kac and these later constructions only worked for particles in $1+1$ dimensions .

Indeed, the process considered in [33-36] is part of a larger class, in fact, by Lorentz boosts new processes can be obtained with particles moving at the speed of light (a simple consequence of the fact that a light-speed particle in an inertial frame is also light-speed in any other inertial frame). The processes of this larger class have in general an unbalanced 
probability rate of velocity inversion i.e., the inversions from right to left occur with a different probability rate of those from left to right, as a consequence, the particle may have a non vanishing average velocity.

The class of these one-dimensional light-speed processes was further extended by considering inversion rates which not only depend on the sign of the velocity but also on position and time. This extension gave the possibility to reformulate the quantum mechanics of a relativistic particle in terms of stochastic processes [37] in the spirit of Nelson's stochastic mechanics [38]. Again, this construction was limited to $1+1$ dimensions.

In this paper we consider a family of processes which generalizes the Kac approach to the $(3+1)$-dimensional case (three space dimensions + time). The goal is to construct a Brownian motion which is the most similar to the Wiener process among all those processes which do not conflict with relativity. We assume that the particle only moves at the speed of light $c$ which implies that velocity can be represented by a point on the surface of a sphere of radius $c$. We also assume that in the 'rest frame', the velocity performs a isotropic Wiener process on that surface (which corresponds to anisotropic Wiener processes in general frames).

In this way the speed is always $c$ which is the largest among those compatible with relativity, but velocity direction changes. It should be remarked the trajectories of the velocity are almost everywhere continuous but they are not differentiable, on the contrary the trajectories of the positions are continuous and differentiable.

In the 'rest frame' the particle is ballistic at short times (position changes proportionally to time), and ordinarly diffusive $\left(E\left[x^{2}\right] \sim t\right)$ at large times for which the average velocity vanishes. Then, one has to consider all the processes generated by Lorentz boosts. The instantaneous velocity of these processes must be also luminal, because a luminal particle is luminal in any inertial frame. Therefore, the velocity still remains on the surface of the sphere, nevertheless, its diffusion is anisotropic and the average velocity is unvanishing at large times. The construction of this family of processes, which transform one into the other by Lorentz boost, needs Ito calculus which leeds to the core equation (10) which represents the entire family.

The paper is simply organized: in Sect. 2 we introduce the process in the 'rest frame'. The velocity process on the sphere is formulated in a new and more economic way which allow a simpler use of Ito calculus. In Sect. 3 we characterize the entire family of processes generated by Lorentz boosts. Nevertheless, the very long application of Ito calculus to reach this goal is postponed in an Appendix that eventually the reader can skip. Averages are computed in Sect. 4 where we also highlight and describe the short-term ballistic behavior versus the long-term diffusive behavior of the particles in the 3D physical space. Summary and outlook can be finally found in Sect. 5 .

\section{Stochastic Equations for the Process in the 'Rest Frame'}

The particle velocity performs a isotropic Wiener process on the surface of a sphere of radius $c$, In this way, while the velocity direction changes, the speed always equals $c$, which is the largest among those compatible with relativity.

The equations governing this process (Ito notation) are:

$$
\begin{aligned}
d \mathbf{x}(t) & =\mathbf{c}(t) d t \\
d \mathbf{c}(t) & =-\omega^{2} \mathbf{c}(t) d t+\omega c d \mathbf{w}(t)
\end{aligned}
$$


where, according to Ito, $d \mathbf{c}(t)=\mathbf{c}(t+d t)-\mathbf{c}(t)$ and $d \mathbf{w}(t)=\mathbf{w}(t+d t)-\mathbf{w}(t)$ is a two component standard Wiener increment on the plane perpendicular to $\mathbf{c}(t)$ such that $E\left[|d \mathbf{w}(t)|^{2}\right]=2 d t$. The only paramether is $\omega$, which has the dimension of the inverse of the square root of a time.

The second of the above equations, given that $d \mathbf{w}(t)$ is a two component Wiener increment tangent to the surface, describes a isotropic Wiener process on that surface of a sphere. This process was studied for the first time at least 70 years ago $[39,40]$.

It is straightforward to verify that $|\mathbf{c}(t)|=c$ at any time $t \geq 0$. In fact, according to Ito, one trivially obtains $d \mathbf{c}^{2}(t)=0$ (in next section this equality is explicitly proven for the general family of processes generated by Lorentz boosts). Therefore, Eq. (1) describes a particle which has constant speed and whose velocity changes direction following continuous but not differentiable trajectories.

The present model can be seen as a 3 space dimensions version of the Kac process. In fact, a constant speed process in one space dimension can be only constructed by considering jumps between the two possible velocities. In three space dimensions, constant speed means that velocity is represented by a point on the surface of a sphere (with the speed as radius). A process over this surface can be realized by allowing jumps between points (velocity jumps from one value to another) or by continuous Wiener trajectories on the surface (velocity changes direction in a continuous way). There are only these two choices for a Markovian generalization of the Kac process to three space dimensions, we followed the second. Nevertheless, the present model can be also considered as a peculiar Ornstein-Uhlenbeck process constructed in such a way that speed remains constant.

In the following pages we will omit the time as an explicit argument when it is not strictly necessary. For example, we will simply write $\mathbf{c}, d \mathbf{c}, \mathbf{w}$ and $d \mathbf{w}$ for $\mathbf{c}(t), d \mathbf{c}(t), \mathbf{w}(t)$ and $d \mathbf{w}(t)$.

The increment $d \mathbf{w}$ has to be a two component Wiener differential perpendicular to $\mathbf{c}$ (which means tangent to the surface), nevertheless, the recipe for its construction is not univocal.

In the early seventies of last century Strook and then Ito $[41,42]$ constructed the increment $d \mathbf{w}$ in the second of Eq. (1) as

$$
d \mathbf{w}=\left(\mathbb{I}-\mathbf{n n}^{\top}\right) d \mathbf{W}
$$

where $\mathbf{n}(t)=\mathbf{c}(t) / c$ is a time dependent unitary vector, $\mathbf{W}$ is a standard three dimensional Wiener process, II is the $3 \times 3$ identity matrix and the row vector $\mathbf{n}^{\top}$ is the transposed of the column vector $\mathbf{n}$. One gets $d \mathbf{c}=-\omega^{2} \mathbf{c} d t+\sigma d \mathbf{W}$ where $\sigma=\omega c\left(\mathbb{I}-\mathbf{n n}^{\top}\right)$ is a $3 \times 3$ matrix.

In the eighties a simpler choice was considered [43,44]:

$$
d \mathbf{w}=\mathbf{n} \times d \mathbf{W},
$$

which leads to $d \mathbf{c}=-\omega^{2} \mathbf{c} d t+\hat{\sigma} d \mathbf{W}$ where $\hat{\sigma}=\omega c[\mathbf{n}]$ with [n] being the $3 \times 3$ skew matrix representation of the vector $\mathbf{n}$. It is easy to check that $\sigma \sigma^{\top}=\hat{\sigma} \hat{\sigma}^{\top}=\omega^{2} c^{2}\left(\mathbb{I}-\mathbf{n n}^{\top}\right)$ which implies that the Forward Kolmogorov Equation is the same for choices (2) and (3). See $[45,46]$ for properties and applications.

Both implementations of the two-dimensional increment $d \mathbf{w}$ are made by a three dimensional Wiener process $\mathbf{W}(t)$, which is somehow redundant for the construction of a two-dimensional increment.

We propose here to use in place of the standard three-components Wiener process $\mathbf{W}=$ $\left(W_{1}, W_{2}, W_{3}\right.$ ), a standard two-components Wiener processes $w_{2}, w_{3}$ (we write $w_{2}, w_{3}$ in place of $W_{2}, W_{3}$ to avoid confusion). Our choice is

$$
d \mathbf{w}=\mathbf{n}_{2} d w_{2}+\mathbf{n}_{3} d w_{3}
$$


where $\mathbf{n}_{\mathbf{2}}(t)$ and $\mathbf{n}_{\mathbf{3}}(t)$ are two unitary vectors perpendicular each other and also perpendicular to $\mathbf{n}(t)$. The Wiener increments are independent which implies $E\left[d w_{1}(t) d w_{2}(t)\right]=0$ and they are standard which means $E\left[(d \mathbf{w}(t))^{2}\right]=2 d t$.

It must be clear that the orientation of $\mathbf{n}_{\mathbf{2}}(t)$ and $\mathbf{n}_{\mathbf{3}}(t)$ can be arbitrarily chosen on the plane perpendicular to $\mathbf{n}(t)$. In the following we make a choice which is motivated by the fact that it is the simplest for our goal, which is to construct, by Ito calculus, the general family of processes generated by Lorentz boosts. In [22] we made a totally different choice which allowed us to separate the space variables from the velocity variables in order to write down a Forward Kolmogorov Equation directely in a 3D configuration space.

Given a constant vector $\mathbf{v}$, we chose here

$$
\mathbf{n}_{\mathbf{2}}=\frac{\mathbf{v} \times \mathbf{n}}{|\mathbf{v} \times \mathbf{n}|}, \quad \mathbf{n}_{3}=\mathbf{n} \times \mathbf{n}_{\mathbf{2}},
$$

so that $\mathbf{v}, \mathbf{n}=\mathbf{c} / \mathrm{c}$ and $\mathbf{n}_{\mathbf{3}}$ are on the same plane and $\mathbf{n}_{\mathbf{2}}$ is perpendicular to it. To fix the ideas one can put the north pole in the $\mathbf{v}$ direction with respect to the center of the sphere, so that $\mathbf{n}_{\mathbf{3}}$ is tangent to a meridian and points to north, while $\mathbf{n}_{\mathbf{2}}$ is tangent to a parallel and points to est. In this way it is simple to pass to spherical coordinates. At the poles (where $\mathbf{n}$ equals \pm $\mathbf{v} /|\mathbf{v}|)$ the unitary vectors $\mathbf{n}_{\mathbf{2}}$ and $\mathbf{n}_{\mathbf{3}}$ can be arbitrarily chosen perpendicularly to $\mathbf{v}$.

Equations (2), (3) and (4) are three totally equivalent way to construct the same Wiener increment tangent to the surface. According to our representation (4), the second equation in (1) rewrites as:

$$
d \mathbf{c}=-\omega^{2} \mathbf{c} d t+\omega c\left(\mathbf{n}_{2} d w_{2}+\mathbf{n}_{3} d w_{3}\right)
$$

the advantage being that we use only a two component Wiener process in place of a three component one, moreover this stochastic equation is straightforwardly associated to the velocity spherical Laplacian in the Kolmogorov Equations when it is expressed in terms of longitude and latitude.

We stress again that our specific choice (5) is only dictated by convenience for later calculations in this paper, any other choice which keeps $\mathbf{n}_{\mathbf{2}}$ and $\mathbf{n}_{\mathbf{3}}$ perpendicular to $\mathbf{n}$ and perpendicular each other is equally admissible.

\section{Lorentz Boosts and Stochastic Equations in a Generic Inertial Frame}

In the 'rest frame' the velocity $\mathbf{c}(t)$ of the particle evolves according to Eq. (6) where $\mathbf{n}_{2}$ and $\mathbf{n}_{\mathbf{3}}$ are defined by (5). Then, assume that this 'rest frame' moves at constant velocity $\mathbf{u}$ (without rotating) with respect to a second inertial frame. Since the choice of $\mathbf{v}$ in (5) is arbitrary, we can leave it to coincide with $\mathbf{u}$. In the next we will only use $\mathbf{v}$ to indicate both the velocity in (5) and the velocity of the 'rest frame'.

According to special relativity, the velocity $\mathbf{c}^{\prime}(t)$ of the particle in the second frame is

$$
\mathbf{c}^{\prime}(t)=\frac{1}{1+\frac{\mathbf{v} \cdot \mathbf{c}(t)}{c^{2}}}\left[\alpha \mathbf{c}(t)+\mathbf{v}+(1-\alpha) \frac{\mathbf{v} \cdot \mathbf{c}(t)}{v^{2}} \mathbf{v}\right]
$$

where $\mathbf{v}, c$ and $\alpha=\left(1-\frac{v^{2}}{c^{2}}\right)^{\frac{1}{2}}$ are constant. Notice that in the above equation the argument of $\mathbf{c}^{\prime}$ is still $t$ and not the time $t^{\prime}$ of the second frame.

By special relativity the velocity in this second inertial frame is also luminal $\left(\left|\mathbf{c}^{\prime}\right|=c\right)$ (at the end of this section we will show that indeed $\left(\mathbf{c}^{\prime}\right)^{2}=\mathbf{c}^{2}=c^{2}$ ). If one also takes into 
account that the time increment $d t^{\prime}$ in the second frame satisfies

$$
\frac{d t}{d t^{\prime}}=\frac{1}{\alpha}\left(1-\frac{\mathbf{v} \cdot \mathbf{c}^{\prime}}{c^{2}}\right),
$$

one should be able to write from (7) and (8) a stochastic equation for $\mathbf{c}^{\prime}\left(t^{\prime}\right)$ analogous to (6) and (5). Notice that in the new equation the time will be $t^{\prime}$ and the increment $d \mathbf{c}^{\prime}=$ $\mathbf{c}^{\prime}\left(t^{\prime}+d t^{\prime}\right)-\mathbf{c}^{\prime}\left(t^{\prime}\right)$ must be expressed in terms of $\mathbf{c}^{\prime}\left(t^{\prime}\right), d t^{\prime}$ and of the increments $d w_{2}^{\prime}=$ $w_{2}\left(t^{\prime}+d t^{\prime}\right)-w_{2}\left(t^{\prime}\right)$ and $d w_{3}^{\prime}=w_{3}\left(t^{\prime}+d t^{\prime}\right)-w_{3}\left(t^{\prime}\right)$. In the second frame the particle will still instantaneously move at the speed of light but, contrarily to the case of the process in the 'rest frame', its average velocity will not vanish at large times.

We define $\delta \mathbf{c}^{\prime}=\mathbf{c}^{\prime}(t+d t)-\mathbf{c}^{\prime}(t)$ (notice the difference with $\left.d \mathbf{c}^{\prime}=\mathbf{c}^{\prime}\left(t^{\prime}+d t^{\prime}\right)-\mathbf{c}^{\prime}\left(t^{\prime}\right)\right)$, then a long and tedious application of Ito calculus (see the Appendix) leads to

$$
\delta \mathbf{c}^{\prime}=-\frac{\omega^{2}}{\alpha^{2}}\left[1-\frac{\mathbf{v} \cdot \mathbf{c}^{\prime}}{c^{2}}\right]^{2} \mathbf{c}^{\prime} d t+\frac{\omega c}{\alpha}\left(1-\frac{\mathbf{v} \cdot \mathbf{c}^{\prime}}{c^{2}}\right)\left(\mathbf{n}_{\mathbf{2}}^{\prime} d w_{2}+\mathbf{n}_{\mathbf{3}}^{\prime} d w_{3}\right)
$$

where $\mathbf{n}_{\mathbf{2}}^{\prime}$ and $\mathbf{n}_{\mathbf{3}}^{\prime}$ are the two unitary vectors perpendicular to $\mathbf{c}^{\prime}$ defined as in (5) (with $\mathbf{n}, \mathbf{n}_{\mathbf{2}}$ and $\mathbf{n}_{\mathbf{3}}$ replaced by $\mathbf{n}^{\prime}=\mathbf{c}^{\prime} / c, \mathbf{n}_{\mathbf{2}}^{\prime}$ and $\mathbf{n}_{\mathbf{3}}^{\prime}$ ). Then, taking into account (8) and remembering that $d w_{3} / d w_{3}^{\prime}=d w_{2} / d w_{2}^{\prime}=\left(d t / d t^{\prime}\right)^{\frac{1}{2}}$ one gets

$$
d \mathbf{c}^{\prime}=-\frac{\omega^{2}}{\alpha^{3}}\left[1-\frac{\mathbf{v} \cdot \mathbf{c}^{\prime}}{c^{2}}\right]^{3} \mathbf{c}^{\prime} d t^{\prime}+\omega c\left[\frac{1}{\alpha}\left(1-\frac{\mathbf{v} \cdot \mathbf{c}^{\prime}}{c^{2}}\right)\right]^{\frac{3}{2}} d \mathbf{w}^{\prime}
$$

where $d \mathbf{w}^{\prime}=\mathbf{n}_{\mathbf{2}}^{\prime} d w_{2}^{\prime}+\mathbf{n}_{\mathbf{3}}^{\prime} d w_{3}^{\prime}$ is a two component increment perpendicular to $\mathbf{c}^{\prime}$ in complete analogy with the process in the 'rest frame'. Notice that by (7) the three vectors $\mathbf{v}, \mathbf{c}$ and $\mathbf{c}^{\prime}$ are on the same plane so that $\mathbf{n}_{\mathbf{3}}, \mathbf{n}_{\mathbf{3}}^{\prime}$ also are on the same plane. As a consequence $\mathbf{n}_{\mathbf{2}}$ and $\mathbf{n}_{\mathbf{2}}^{\prime}$ are both perpendicular to that plane so that $\mathbf{n}_{\mathbf{2}}=\mathbf{n}_{\mathbf{2}}^{\prime}$.

This is the core equation, each process of the family is labeled by the index $\mathbf{v}$, the case $\mathbf{v}=0$ corresponds to the process in the 'rest frame'.

One can easily prove from (6) that the speed of the particle remains constantly luminal i.e., $d\left|\mathbf{c}^{\prime}(t)\right|=0$, in fact by Ito calculus

$$
\begin{aligned}
d\left|\mathbf{c}^{\prime}\right|^{2}= & -2 \frac{\omega^{2}}{\alpha^{3}}\left[1-\frac{\mathbf{v} \cdot \mathbf{c}^{\prime}}{c^{2}}\right]^{3}\left|\mathbf{c}^{\prime}\right|^{2} d t^{\prime}+2 c^{2} \frac{\omega^{2}}{\alpha^{3}}\left[1-\frac{\mathbf{v} \cdot \mathbf{c}^{\prime}}{c^{2}}\right]^{3} d t^{\prime} \\
& +2 \frac{c \omega^{3}}{\alpha^{3}}\left[1-\frac{\mathbf{v} \cdot \mathbf{c}^{\prime}}{c^{2}}\right]^{3} \mathbf{c}^{\prime} \cdot d \mathbf{w}^{\prime}
\end{aligned}
$$

where the second term at the right comes from the second order contribution to Ito differential. Since $\mathbf{c}^{\prime}$ and $d \mathbf{w}^{\prime}$ are perpendicular the equation reduces to

$$
d\left|\mathbf{c}^{\prime}\right|^{2}=-2 \frac{\omega^{2}}{\alpha^{3}}\left[1-\frac{\mathbf{v} \cdot \mathbf{c}^{\prime}}{c^{2}}\right]^{3}\left(\left|\mathbf{c}^{\prime}\right|^{2}-c^{2}\right) d t^{\prime}=0
$$

where the last equality holds if the initial velocity is luminal i.e., $|\mathbf{c}(0)|=c$.

The fact that the process remains luminal is not astonishing since a particle moving at the speed of light also moves at the speed of light in any other inertial frame. Therefore, Eq. (10) defines a family of light-speed processes (indexed by $\mathbf{v}$ ) which transform one in the other by Lorentz boost.

We already mentioned that in a generic inertial frame (indexed by $\mathbf{v}$ ) the particle has a long term unvanishing average velocity. This is simple consequence of the fact that the rest frame moves at velocity $\mathbf{v}$ with respect to the generic frame and of the fact that the long term 
average velocity vanishes for the process in the 'rest frame' (we will prove this in the next section).

The reason of this long term average lies in the fact that the diffusion of $\mathbf{c}^{\prime}$ slows down the more $\mathbf{v} \cdot \mathbf{c}^{\prime}$ is large as it can be inferred from Eq. (10), This means that the particle spends more time with values of $\mathbf{c}^{\prime}$ aligned with $\mathbf{v}$ and less time when it is anti-aligned. This is exactly the same one has with the $(1+1)$-dimensional Kac process associated to the telegrapher equation. In that simpler case a non vanishing average velocity is determined by the fact that unbalanced rates of inversions lead to a longer permanence of the velocity in one of the two directions.

\section{Short-Term Ballistic Behavior Versus Long-Term Diffusive Behavior}

In this section we only consider the process in the 'rest frame', all results concerning averages can be eventually Lorentz transformed for the processes in a generic inertial frame.

The stochastic equation (1) can be recast in an integral equation:

$$
\begin{aligned}
& \mathbf{x}(t)=\mathbf{x}(0)+\int_{0}^{t} \mathbf{c}(s) d s, \\
& \mathbf{c}(t)=e^{-\omega^{2} t}\left[\mathbf{c}(0)+\omega c \int_{0}^{t} e^{\omega^{2} s} d \mathbf{w}(s)\right] .
\end{aligned}
$$

This is not a solution because $d \mathbf{w}(s)$, according to (4) and (5), depends on $\mathbf{c}(t)$. Let us mention that the proof that the particle velocity remains constantly luminal i.e., $|\mathbf{c}(t)|=c$ can be eventually also obtained by the second integral equation in (13).

Starting from the second integral equation in (13) one can easily find out that the following averages hold for $t \geq s \geq 0$ :

$$
\begin{aligned}
& E[\mathbf{c}(t)]=e^{-\omega^{2} t} \mathbf{c}(0), \\
& E[\mathbf{c}(t) \cdot \mathbf{c}(s)]=c^{2} e^{-\omega^{2}(t+s)}+2 \omega^{2} c^{2} e^{-\omega^{2}(t+s)} \int_{0}^{s} e^{2 \omega^{2} u} d u=c^{2} e^{-\omega^{2}(t-s)} .
\end{aligned}
$$

Notices that the first of the equalities above says that the average velocity $E[\mathbf{c}(t)]$ vanishes for large $t$ however, for a generic inertial frame, $E\left[\mathbf{c}^{\prime}\left(t^{\prime}\right)\right]$ doesn't vanish for large $t^{\prime}$. This is simple consequence of the fact that the rest frame moves at velocity $\mathbf{v}$ with respect to the generic frame.

Using these averages and the first of the integral equations in (13), one also obtains

$$
\begin{aligned}
& E[\mathbf{x}(t)]=\mathbf{x}(0)+\frac{1-e^{-\omega^{2} t}}{\omega^{2}} \mathbf{c}(0), \\
& E\left[(\mathbf{x}(t)-\mathbf{x}(0))^{2}\right]=2 c^{2} \int_{0}^{t} \int_{0}^{s} e^{-\omega^{2}(s-u)} d u d s=\frac{2 c^{2}}{\omega^{2}} t-\frac{2 c^{2}}{\omega^{4}}\left(1-e^{-\omega^{2} t}\right) .
\end{aligned}
$$

The above averages imply, for large times, a diffusive behavior with coefficient $\frac{c^{2}}{\omega^{2}}$, in this limit one has in fact

$$
E\left[(\mathbf{x}(t)-\mathbf{x}(0))^{2}\right] \sim \frac{2 c^{2}}{\omega^{2}} t,
$$

on the contrary, for short times one has

$$
E[\mathbf{x}(t)-\mathbf{x}(0)] \sim \mathbf{c}(0) t, \quad E\left[(\mathbf{x}(t)-\mathbf{x}(0))^{2}\right] \sim c^{2} t^{2},
$$


which means ballistic behavior at the speed of light.

The short-term ballistic behavior it is not completely unexpected. In fact, for a small time $\Delta t \ll 1 / \omega^{2}$ the velocity of a particle remains almost constant. This can be understood from the second equation in (1) which, having defined $\Delta \mathbf{c}=\mathbf{c}(\Delta t)-\mathbf{c}(0)$, implies $|\Delta \mathbf{c}| \approx$ $\left|\omega^{2} \mathbf{c} \Delta t-\omega c \mathbf{w}(\Delta t)\right| \leq \omega^{2} c \Delta t+\omega c|\mathbf{w}(\Delta t)|$. Since $|\mathbf{w}(\Delta t)|$ is of the order of $\sqrt{\Delta t}$ and given that $\Delta t \ll 1 / \omega^{2}$, one finally obtains $|\Delta \mathbf{c}| / c \ll 1$. This short-term ballistic behavior was already observed and described in depth in [47] for the Relativistic Ornstein-Uhlenbeck Process [4]. In case, it could be also coarsely derived from Eq. (2) in [47] by adapting the line of reasoning we followed above.

\section{Summary and Outlook}

In conclusion we have found that Eq. (10) describes a family of light-speed processes which transform one in the other by Lorentz boost. Their main characteristics can be resumed as follows:

- The family of processes that we propose generalizes to 3+1 dimensions the 1956 idea of Mark Kac in the sense that particles only move at the speed of light. Although the Kac process can be generalized to $3+1$ dimensions in a different way, for example considering a velocity which performs jumps in place of having continuous trajectories, we think the process presented here, having as a constitutive ingredient the Wiener process, is the most natural choice for this generalization. Moreover, since the speed is always the maximum possible given the relativistic constraint, it posseses the trajectories which better mimics the (infinite speed) Wiener trajectories;

- The long term average velocity vanishes in the 'rest frame', but it does not in a generic frame. This is is a consequence of the fact that according to (10) the diffusion of the velocity slows down the more $\mathbf{v} \cdot \mathbf{c}^{\prime}$ is large. In turn, this means that the particle spends more time with values of $\mathbf{c}^{\prime}$ aligned with $\mathbf{v}$ and less time when it is anti-aligned. This is exactly the same situation one has with the $(1+1)$-dimensional Kac process associated to the telegrapher equation since the probability rate of inversion of velocity can be different for left/right and right/left inversions [37];

- For large times the behavior of the position is diffusive with coefficient $\frac{c^{2}}{\omega^{2}}$, one has in fact $E\left[(\mathbf{x}(t)-\mathbf{x}(0))^{2}\right] \sim \frac{2 c^{2}}{\omega^{2}} t$. On the contrary, for short times $E[\mathbf{x}(t)-\mathbf{x}(0)] \sim c t$ and $E\left[(\mathbf{x}(t)-\mathbf{x}(0))^{2}\right] \sim c^{2} t^{2}$ which means ballistic behavior at the speed of light. The short-term ballistic behavior holds for $t$ smaller than $1 / \omega^{2}$.

This process is the natural candidate for modeling the Brownian motion of mass-less particles, nevertheless, its use should not be limited to this case. The situation is similar in the non-relativistic realm; thought a particle with infinite speed is unphysical, the Wiener process is largely used to model its erratic movement. Another point that deserves investigation and which contributed to prompt this work is the possible connection of its Backward Kolmgorov Equation with relativistic equations as Klein-Gordon and Dirac. The goal would be to find a generalization of the Gaveau et al. approach to the (3+1)-dimensional case. This topic is presently under study.

Funding Open access funding provided by Università degli Studi dell' Aquila within the CRUI-CARE Agreement. 
Open Access This article is licensed under a Creative Commons Attribution 4.0 International License, which permits use, sharing, adaptation, distribution and reproduction in any medium or format, as long as you give appropriate credit to the original author(s) and the source, provide a link to the Creative Commons licence, and indicate if changes were made. The images or other third party material in this article are included in the article's Creative Commons licence, unless indicated otherwise in a credit line to the material. If material is not included in the article's Creative Commons licence and your intended use is not permitted by statutory regulation or exceeds the permitted use, you will need to obtain permission directly from the copyright holder. To view a copy of this licence, visit http://creativecommons.org/licenses/by/4.0/.

\section{Appendix: Ito Calculus}

In this appendix we apply Ito calculus, in order to obtain Eq. (9) from Eq. (7). Since we defined $\delta \mathbf{c}^{\prime}=\mathbf{c}^{\prime}(t+d t)-\mathbf{c}^{\prime}(t)$, then from (7) we immediately get

$$
\begin{aligned}
\delta \mathbf{c}^{\prime}= & \frac{1}{1+\frac{\mathbf{v} \cdot(\mathbf{c}+d \mathbf{c})}{c^{2}}}\left[\alpha(\mathbf{c}+d \mathbf{c})+\mathbf{v}+(1-\alpha) \frac{\mathbf{v} \cdot(\mathbf{c}+d \mathbf{c})}{v^{2}} \mathbf{v}\right] \\
& -\frac{1}{1+\frac{\mathbf{v} \cdot \mathbf{c}}{c^{2}}}\left[\alpha \mathbf{c}+\mathbf{v}+(1-\alpha) \frac{\mathbf{v} \cdot \mathbf{c}}{v^{2}} \mathbf{v}\right]
\end{aligned}
$$

where $\mathbf{c}+d \mathbf{c}=\mathbf{c}(t+d t)$ and $\mathbf{c}=\mathbf{c}(t)$. This is still not a Ito increment, but it is the trivial application of the definition $\delta \mathbf{c}^{\prime}=\mathbf{c}^{\prime}(t+d t)-\mathbf{c}^{\prime}(t)$. This equation can be exactly rewritten as

$$
\begin{aligned}
\delta \mathbf{c}^{\prime}= & \frac{d \epsilon}{1+d \epsilon}\left[(1-\alpha) \frac{c^{2}}{v^{2}} \mathbf{v}-\frac{1}{1+\frac{\mathbf{v} \cdot \mathbf{c}}{c^{2}}}\left(\alpha \mathbf{c}+\mathbf{v}+(1-\alpha) \frac{\mathbf{v} \cdot \mathbf{c}}{v^{2}} \mathbf{v}\right)\right] \\
& +\frac{1}{1+d \epsilon} \frac{\alpha d \mathbf{c}}{1+\frac{\mathbf{v} \cdot \mathbf{c}}{c^{2}}}
\end{aligned}
$$

where $d \mathbf{c}$ is given by (6) and where

$$
d \epsilon=\frac{1}{1+\frac{\mathbf{v} \cdot \mathbf{c}}{c 2}} \frac{\mathbf{v} \cdot d \mathbf{c}}{c^{2}}=-\frac{\omega^{2}}{c^{2}} \frac{\mathbf{v} \cdot \mathbf{c}}{1+\frac{\mathbf{v} \cdot \mathbf{c}}{c 2}} d t+\frac{\omega}{c} \frac{\mathbf{v} \cdot \mathbf{n}_{\mathbf{3}}}{1+\frac{\mathbf{v} \cdot \mathbf{c}}{c 2}} d w_{3} .
$$

The next step is to calculate the right side of the Eq. (19) in terms of the new variables $\mathbf{c}^{\prime}$, $\mathbf{n}_{\mathbf{2}}^{\prime}$ and $\mathbf{n}_{\mathbf{3}}^{\prime}$. First of all, using (7) we immediately rewrite the Eq. (19) as

$$
\delta \mathbf{c}^{\prime}=\frac{1}{1+d \epsilon}\left[d \epsilon\left((1-\alpha) \frac{c^{2}}{v^{2}} \mathbf{v}-\mathbf{c}^{\prime}\right)+\frac{1-\frac{\mathbf{v} \cdot \mathbf{c}^{\prime}}{c^{2}}}{\alpha} d \mathbf{c}\right],
$$

but we also need to calculate $d \epsilon$ and $d \mathbf{c}$ in terms of the new coordinates. In order to reach this goal we need to recall that

$$
\mathbf{c}=\frac{1}{1-\frac{\mathbf{v} \cdot \mathbf{c}^{\prime}}{c^{2}}}\left[\alpha \mathbf{c}^{\prime}-\mathbf{v}+(1-\alpha) \frac{\mathbf{v} \cdot \mathbf{c}^{\prime}}{v^{2}} \mathbf{v}\right] \rightarrow \mathbf{v} \cdot \mathbf{c}=\frac{1}{1-\frac{\mathbf{v} \cdot \mathbf{c}^{\prime}}{c^{2}}}\left[\mathbf{v} \cdot \mathbf{c}^{\prime}-v^{2}\right]
$$

moreover

$$
\begin{aligned}
c \mathbf{n}_{3} & =\frac{1}{1-\frac{\mathbf{v} \cdot \mathbf{c}^{\prime}}{c^{2}}}\left[\alpha c \mathbf{n}_{\mathbf{3}}^{\prime}-\mathbf{v} \times \mathbf{n}_{\mathbf{2}}^{\prime}+(1-\alpha) \frac{\mathbf{v} \cdot \mathbf{c}^{\prime}}{v^{2}} \mathbf{v} \times \mathbf{n}_{\mathbf{2}}^{\prime}\right] \rightarrow \mathbf{v} \cdot \mathbf{n}_{\mathbf{3}} \\
& =\alpha \frac{\mathbf{v} \cdot \mathbf{n}_{\mathbf{3}}^{\prime}}{1-\frac{\mathbf{v} \cdot \mathbf{c}^{\prime}}{c^{2}}} .
\end{aligned}
$$


From these two last equations one easily realize that the three vectors $\mathbf{c}, \mathbf{v}$ and $\mathbf{c}^{\prime}$ are co-planar. As well, $\mathbf{n}_{3}$ and $\mathbf{n}_{\mathbf{3}}^{\prime}$ lie on the same plane. Moreover, $\mathbf{n}_{\mathbf{2}}^{\prime}=\mathbf{n}_{\mathbf{2}}$ is perpendicular to that plane. We get

$$
d \epsilon=-\frac{\omega^{2}}{c^{2}} \frac{\mathbf{v} \cdot \mathbf{c}^{\prime}-v^{2}}{\alpha^{2}} d t+\frac{\omega}{c} \frac{\mathbf{v} \cdot \mathbf{n}^{\prime}}{\alpha} d w_{3},
$$

where $d \mathbf{c}$ is given by (6) with $\mathbf{n}_{\mathbf{2}}=\mathbf{n}_{\mathbf{2}}^{\prime}$ and $\mathbf{c}$ and $\mathbf{n}_{\mathbf{3}}$ given respectively by the first equation in (22) and the first equation in (23).

We are now ready compute the Ito differential i.e., we are ready to rewrite the differential $\delta \mathbf{c}^{\prime}$ keeping only terms of order $d t$. To obtain this result we have first of all to expand (21) to the second order with respect to the differentials:

$$
\begin{aligned}
\delta \mathbf{c}^{\prime} \approx & {\left[d \epsilon\left((1-\alpha) \frac{c^{2}}{v^{2}} \mathbf{v}-\mathbf{c}^{\prime}\right)+\frac{1-\frac{\mathbf{v} \cdot \mathbf{c}^{\prime}}{c^{2}}}{\alpha} d \mathbf{c}\right] } \\
& \left.-\left[(d \epsilon)^{2}\left((1-\alpha) \frac{c^{2}}{v^{2}} \mathbf{v}-\mathbf{c}^{\prime}\right)+d \epsilon d \mathbf{c}\right] \frac{1-\frac{\mathbf{v} \cdot \mathbf{c}^{\prime}}{c^{2}}}{\alpha}\right],
\end{aligned}
$$

then, we have replace the second order differentials $(d \epsilon)^{2}$ and $d \epsilon d \mathbf{c}$ by the terms proportional to $d t$ of their averages:

$$
(d \epsilon)^{2} \approx\left(\frac{\omega}{c}\right)^{2} \frac{\left(\mathbf{v} \cdot \mathbf{n}_{3}^{\prime}\right)^{2}}{\alpha^{2}} d t, \quad d \epsilon d \mathbf{c} \approx \frac{\omega^{2}}{\alpha}\left(\mathbf{v} \cdot \mathbf{n}_{\mathbf{3}}^{\prime}\right) \mathbf{n}_{\mathbf{3}} d t
$$

where $\mathbf{n}_{\mathbf{3}}$ must be expressed in terms of the new variables by the first equation in (23).

Let us rewrite Eq. (25) as

$$
\delta \mathbf{c}^{\prime}=\delta \mathcal{A}+\delta \mathcal{B}+\delta \mathcal{C}
$$

where $\delta \mathcal{A}$ is the term proportional to $d t$ which comes from the first order differentials (the deterministic part of the first term between square parenthesis in $(25)), \delta \mathcal{B}$ is the term proportional to $d t$ which comes from the second order differential (the second term between square parenthesis in (25)) and $\delta \mathcal{C}$ is the random term (the random part of the first term between square parenthesis in (25)). After having expressed all the old variables in terms of the new ones (except $\mathbf{n}_{3}$ ), we have

$$
\begin{aligned}
& \delta \mathcal{A}=-\left[\frac{\omega^{2}}{c^{2}} \frac{\mathbf{v} \cdot \mathbf{c}^{\prime}-v^{2}}{\alpha^{2}}\left((1-\alpha) \frac{c^{2}}{v^{2}} \mathbf{v}-\mathbf{c}^{\prime}\right)+\frac{\omega^{2}}{\alpha}\left(\alpha \mathbf{c}^{\prime}-\mathbf{v}+(1-\alpha) \frac{\mathbf{v} \cdot \mathbf{c}^{\prime}}{v^{2}} \mathbf{v}\right)\right] d t, \\
& \delta \mathcal{B}=-\frac{\omega^{2}}{\alpha^{2}}\left(\mathbf{v} \cdot \mathbf{n}_{3}^{\prime}\right)\left[\frac{\mathbf{v} \cdot \mathbf{n}_{3}^{\prime}}{c^{2}}\left((1-\alpha) \frac{c^{2}}{v^{2}} \mathbf{v}-\mathbf{c}^{\prime}\right)+\left(1-\frac{\mathbf{v} \cdot \mathbf{c}^{\prime}}{c^{2}}\right) \mathbf{n}_{3}\right] d t, \\
& \delta \mathcal{C}=\left[\frac{\omega}{c} \frac{\mathbf{v} \cdot \mathbf{n}_{\mathbf{3}}^{\prime}}{\alpha}\left((1-\alpha) \frac{c^{2}}{v^{2}} \mathbf{v}-\mathbf{c}^{\prime}\right) d w_{3}+\frac{1-\frac{\mathbf{v} \cdot \mathbf{c}^{\prime}}{c^{2}}}{\alpha} \omega c\left(\mathbf{n}_{\mathbf{2}}^{\prime} d w_{2}+\mathbf{n}_{\mathbf{3}} d w_{3}\right)\right]
\end{aligned}
$$

with $\mathbf{n}_{\mathbf{3}}$ given by (23) in terms of the new variables. After some rearrangement of terms we get:

$$
\begin{aligned}
\delta \mathcal{A} & =-\left[\frac{\omega^{2}}{c^{2}} \frac{\mathbf{v} \cdot \mathbf{c}^{\prime}}{\alpha^{2}}-\frac{\omega^{2}}{\alpha^{2}}\right] \mathbf{v} d t+\left[\frac{\omega^{2}}{c^{2}} \frac{\mathbf{v} \cdot \mathbf{c}^{\prime}-c^{2}}{\alpha^{2}}\right] \mathbf{c}^{\prime} d t \\
& =\frac{\omega^{2}}{\alpha^{2}}\left[1-\frac{\mathbf{v} \cdot \mathbf{c}^{\prime}}{c^{2}}\right]\left(\mathbf{v}-\mathbf{c}^{\prime}\right) d t
\end{aligned}
$$


This differential lies in the plane of $\mathbf{c}^{\prime}$ and $\mathbf{n}_{\mathbf{3}}^{\prime}$ and can be decomposed along these two vectors:

$$
\delta \mathcal{A}=-\frac{\omega^{2}}{\alpha^{2}}\left[1-\frac{\mathbf{v} \cdot \mathbf{c}^{\prime}}{c^{2}}\right]^{2} \mathbf{c}^{\prime} d t+\frac{\omega^{2}}{\alpha^{2}}\left[1-\frac{\mathbf{v} \cdot \mathbf{c}^{\prime}}{c^{2}}\right]\left(\mathbf{v} \cdot \mathbf{n}_{3}^{\prime}\right) \mathbf{n}_{\mathbf{3}}^{\prime} d t .
$$

Analogously, the term $\delta \mathcal{B}$, after decomposition along $\mathbf{c}^{\prime}$ and $\mathbf{n}_{\mathbf{3}}^{\prime}$, can be rewritten as

$$
\begin{aligned}
\delta \mathcal{B}=- & \frac{\omega^{2}}{\alpha^{2}}\left(\mathbf{v} \cdot \mathbf{n}_{\mathbf{3}}^{\prime}\right)\left(1-\frac{\mathbf{v} \cdot \mathbf{c}^{\prime}}{c^{2}}\right)\left[\frac{\mathbf{v} \cdot \mathbf{n}_{3}^{\prime}}{1-\frac{\mathbf{v} \cdot \mathbf{c}^{\prime}}{c^{2}}}\left((1-\alpha) \frac{\mathbf{v} \cdot \mathbf{c}^{\prime}}{v^{2}}-1\right)+\mathbf{n}_{\mathbf{3}} \cdot \mathbf{c}^{\prime}\right] \frac{\mathbf{c}^{\prime}}{c^{2}} d t \\
& -\frac{\omega^{2}}{\alpha^{2}}\left(\mathbf{v} \cdot \mathbf{n}_{\mathbf{3}}^{\prime}\right)\left(1-\frac{\mathbf{v} \cdot \mathbf{c}^{\prime}}{c^{2}}\right)\left[\frac{\mathbf{v} \cdot \mathbf{n}_{\mathbf{3}}^{\prime}}{\left(1-\frac{\mathbf{v} \cdot \mathbf{c}^{\prime}}{c^{2}}\right)}\left((1-\alpha) \frac{\mathbf{v} \cdot \mathbf{n}_{\mathbf{3}}^{\prime}}{v^{2}}\right)+\mathbf{n}_{\mathbf{3}} \cdot \mathbf{n}_{\mathbf{3}}^{\prime}\right] \\
& \times \mathbf{n}_{\mathbf{3}}^{\prime} d t .
\end{aligned}
$$

Since $\mathbf{c}^{\prime} \cdot \mathbf{n}_{\mathbf{3}}=-\mathbf{c} \cdot \mathbf{n}_{\mathbf{3}}^{\prime}$, from (22) by scalar product with $\mathbf{n}_{\mathbf{3}}^{\prime}$, one gets

$$
\mathbf{c}^{\prime} \cdot \mathbf{n}_{3}=\frac{1}{1-\frac{\mathbf{v} \cdot \mathbf{c}^{\prime}}{c^{2}}}\left[1-(1-\alpha) \frac{\mathbf{v} \cdot \mathbf{c}^{\prime}}{v^{2} c^{2}}\right] \mathbf{v} \cdot \mathbf{n}_{3}^{\prime},
$$

therefore the first term at the right side of equality (31) vanishes, moreover from (22) and by the definitions of $\mathbf{n}_{\mathbf{3}}$ and $\mathbf{n}_{\mathbf{3}}^{\prime}$ one has that

$$
\mathbf{n}_{3} \cdot \mathbf{n}_{3}^{\prime}=\frac{\mathbf{c} \cdot \mathbf{c}^{\prime}}{c^{2}}=\frac{1}{1-\frac{\mathbf{v} \cdot \mathbf{c}^{\prime}}{c^{2}}}\left[\alpha-\frac{\mathbf{v} \cdot \mathbf{c}^{\prime}}{c^{2}}+(1-\alpha) \frac{\left(\mathbf{v} \cdot \mathbf{c}^{\prime}\right)^{2}}{v^{2}}\right],
$$

which, can be substituted in the second term of (31) in order to obtain

$$
\delta \mathcal{B}=-\frac{\omega^{2}}{\alpha^{2}}\left(\mathbf{v} \cdot \mathbf{n}_{\mathbf{3}}^{\prime}\right)\left(1-\frac{\mathbf{v} \cdot \mathbf{c}^{\prime}}{c^{2}}\right) \mathbf{n}_{\mathbf{3}}^{\prime} d t .
$$

A scalar product of the third of (28) with $\mathbf{c}^{\prime}$ gives

$$
\mathbf{c}^{\prime} \cdot \delta \mathcal{C}=\frac{\omega c}{\alpha}\left[\mathbf{v} \cdot \mathbf{n}_{3}^{\prime}\left((1-\alpha) \frac{\mathbf{v} \cdot \mathbf{c}^{\prime}}{v^{2}}-1\right)+\left(1-\frac{\mathbf{v} \cdot \mathbf{c}^{\prime}}{c^{2}}\right)\left(\mathbf{c}^{\prime} \cdot \mathbf{n}_{3}\right)\right] d w_{3}=0
$$

where the equality is obtained using (32). Construction is coherent since the Wiener increment of $\mathbf{c}^{\prime}$ has no component parallel to $\mathbf{c}^{\prime}$ itself. Therefore, by decomposition along $\mathbf{n}_{\mathbf{2}}^{\prime}=\mathbf{n}_{\mathbf{2}}^{\prime}$ and $\mathbf{n}_{\mathbf{3}}^{\prime}$ we have

$$
\delta \mathcal{C}=\frac{\omega c}{\alpha}\left[1-\frac{\mathbf{v} \cdot \mathbf{c}^{\prime}}{c^{2}}\right] \mathbf{n}_{2}^{\prime} d w_{2}+\frac{\omega c}{\alpha}\left[(1-\alpha) \frac{\left(\mathbf{v} \cdot \mathbf{n}_{3}^{\prime}\right)^{2}}{v^{2}}+\left(1-\frac{\mathbf{v} \cdot \mathbf{c}^{\prime}}{c^{2}}\right) \frac{\mathbf{c}^{\prime} \cdot \mathbf{c}}{c^{2}}\right] \mathbf{n}_{\mathbf{3}}^{\prime} d w_{3},
$$

which given (33) can be rewritten as

$$
\delta \mathcal{C}=\frac{\omega c}{\alpha}\left(1-\frac{\mathbf{v} \cdot \mathbf{c}^{\prime}}{c^{2}}\right)\left(\mathbf{n}_{\mathbf{2}}^{\prime} d w_{2}+\mathbf{n}_{\mathbf{3}}^{\prime} d w_{3}\right) .
$$

Finally, by $\delta \mathbf{c}^{\prime}=\delta \mathcal{A}+\delta \mathcal{B}+\delta \mathcal{C}$ we finally obtain

$$
\delta \mathbf{c}^{\prime}=-\frac{\omega^{2}}{\alpha^{2}}\left[1-\frac{\mathbf{v} \cdot \mathbf{c}^{\prime}}{c^{2}}\right]^{2} \mathbf{c}^{\prime} d t+\frac{\omega c}{\alpha}\left(1-\frac{\mathbf{v} \cdot \mathbf{c}^{\prime}}{c^{2}}\right)\left(\mathbf{n}_{\mathbf{2}}^{\prime} d w_{2}+\mathbf{n}_{3}^{\prime} d w_{3}\right),
$$

which is Eq. (9) that we use in Sect. 3 and which allows to find out Eq. (10) which characterizes the general class of the light-speed processes. 


\section{References}

1. Dudley, R.M.: Lorentz-invariant Markov processes in relativistic phase space. Arkiv Mat. 6, 241-268 (1965)

2. Dudley, R.M.: A note on Lorentz-invariant Markov processes. Arkiv Mat. 6, 575-581 (1967)

3. Dudley, R.M.: Asymptotics of some relativistic Markov processes. Proc. Natl. Acad. Sci. USA 70, 35513555 (1973)

4. Debbasch, F., Mallick, K., Rivet, J.P.: Relativistic Ornstein-Uhlenbeck Process. J. Stat. Phys. 88, 945-966 (1997)

5. Barbachoux, C., Debbasch, F., Rivet, J.P.: The spatially one-dimensional relativistic Ornstein-Uhlenbeck process in a $\mathrm{n}$ arbitrary inertial frame. Eur. Phys. J. B 19, 37-47 (2001)

6. Dunkel, J., Hänggi, P.: Theory of the relativistic Brownian motion: the (1+1)-dimensional case. Phys. Rev. E 71, 016124 (2005)

7. Dunkel, J., Hänggi, P.: Theory of the relativistic Brownian motion: the (1+3)-dimensional case. Phys. Rev. E 72, 036106 (2005)

8. Dunkel, J., Talkner, P., Hänggi, P.: Relativistic diffusion processes and random walk models. Phys. Rev. E 75, 043001 (2007)

9. Franchi, J., Le Jan, Y.: it Relativistic diffusions and Schwarzschild geometry. Commun. Pure Appl. Math. 60, 187-251 (2006)

10. Angst, J., Franchi, J.: Central limit theorem for a class of relativistic diffusions. J. Math. Phys. 48, 083101 (2007)

11. Franchi, J.: Relativistic diffusion in Gödels universe. Commun. Math. Phys. 290, 523-555 (2009)

12. Chevalier, C., Debbasch, F.: Relativistic diffusions: a unifying approach. J. Math. Phys. 49, 043303 (2008)

13. Chevalier, C., Debbasch, F.: A unifying approach to relativistic diffusions and H-theorems. Mod. Phys. Lett. B 22, 383-392 (2008)

14. Herrmann, J.: Diffusion in the special theory of relativity. Phys. Rev. E 80, 051110 (2009)

15. Haba, Z.: Relativistic diffusion. Phys. Rev. E 79, 021128 (2009)

16. Haba, Z.: Relativistic diffusion with friction on a pseudo-Riemannian manifold. Class. Quantum Gravity 27, 095021 (2010)

17. Haba, Z.: Energy and entropy of relativistic diffusing particles. Mod. Phys. Lett. A 25, 2683-2695 (2010)

18. Haba, Z.: Non-linear relativistic diffusions. Phys. A 390, 2776-2786 (2011)

19. Haba, Z., Stachowski, A., Szydłowski, M.: Dynamics of the diffusive DM-DE interaction—dynamical system approach. J. Cosmol. Astropart. Phys. 024, 2776-2786 (2016)

20. Bailleul, I.: A stochastic approach to relativistic diffusions. Annales de l'Institut Henri Poincaré, Probabilités et Statistiques 46, 760-795 (2010)

21. Félix, J.A.A.: A relativistic diffusion model in kinetic theory. Ph.D. Thesis, Editor: Universidad de Granada 1-13 (2015)

22. Serva, M.; Random motion of light-speed particles. J. Stat. Phys. 181, 1603-1608 (2020)

23. Scalas, E., Gabriel, A.T., Martin, E., Germano, G.: Velocity and energy distributions in microcanonical ensembles of hard spheres. Phys. Rev. E 92, 022140 (2015)

24. Gleyzes, S., Kuhr, S., Guerlin, C., Bernu, J., Deléglise, S., Hoff, U.B., Brune, M., Raimond, J.-M., Haroche, S.: Quantum jumps of light recording the birth and death of a photon in a cavity. Nature 446, 297-300 (2007)

25. Ichinose, T.: Path integral for the Dirac equation in two space-time dimensions. Proc. Jpn. Acad. 58A, 290-293 (1982)

26. Ichinose, T., Tamura, H.: Propagation of a Dirac particle—a path integral approach. J. Math. Phys. 25, 1810-1819 (1984)

27. Jacobson, T.: Spinor chain path integral for the Dirac equation. Phys. Rev. Lett. 53, 419-422 (1984)

28. Ichinose, T., Tamura, H.: Imaginary-time path integral for a relativistic spinless particle in an electromagnetic field. Commun. Math. Phys. bf 105, 239-257 (1986)

29. Ichinose, T., Tamura, H.: Path integral approach to relativistic quantum mechanics-two-dimensional Dirac equation. Progress Theor. Phys. Suppl. 92, 144-175 (1987)

30. De Angelis, G.F., Serva, M.: On the relativistic Feynman-Kac-Ito formula. J. Phys. A: Math. Gen. 23, L965-L968 (1990)

31. De Angelis, G.F., Rinaldi, A., Serva, M.: Imaginary-time path integral for a relativistic spin-(1/2) particle in a magnetic field. Europhys. Lett. 14, 95-100 (1991)

32. Serva, M.: Relativistic stochastic processes associated to Klein-Gordon equation. Annales de l'Institut Henri Poincaré, Phisique Théorique 49, 415-432 (1988) 
33. Kac, M.: A stochastic model related to the telegrapher's equation. Rocky Mountain Journal of Mathematics 4, 497-510, (1974). Reprinted from Some Stochastic Problems in Physics and Mathematics. Magnolia Petroleum Company Colloquium Lectures in the Pure and Applied Sciences 2 (1956)

34. Gaveau, B., Jacobson, T., Kac, M., Schulman, L.S.: Relativistic extension of the analogy between Quantum Mechanics and Brownian Motion. J. Phys. A: Math. Gen. 17, 2433-2451 (1984)

35. Blanchard, Ph., Combe, Ph., Sirugue, M., Sirugue-Collin, M.: Path integral representation for the solution of the Dirac equation in presence of an electromagnetic field. In: Gutzwiller, M.C. et al. (eds.). Proceedings of the Bibos Conference Path Integrals from mev to Mev. World Scientific, Singapore (1986)

36. Combe, Ph., Sirugue, M., Sirugue-Collin, M.: Point processes and quantum physics: Some recent developments and results. In: Procedings of the 8th International Congress on Mathematical Physics $(\mathrm{M} \cap \Phi)$, Marseille 1986, World Scientific, Singapore, pp. 421-430 (1987)

37. De Angelis, G.F., Jona-Lasinio, G., Serva, M., Zanghi, Nino: Stochastic mechanics of a Dirac particle in two spacetime dimensions. J. Phys. A: Math. Gen. 19, 865-871 (1986)

38. Nelson, E.: Dynamical Theories of Brownian Motion. Princeton University Press, Princeton (1967)

39. Yosida, K.: Brownian motion on the surface of the 3-sphere. Ann. Math. Stat. 20, 292-296 (1949)

40. Yosida, K.: On Brownian motion in a homogeneous Riemannian space. Pacif. J. Math. 2, 263-270 (1952)

41. Stroock, D.: On the growth of stochastic integrals. Z. Wahrsch. verw. Gebiete 18, 340-344 (1971)

42. Itô, K.: Stochastic calculus. In: Proceedings of the International Symposium on Mathematical Problems in Theoretical Physics (Kyoto). Lecture Notes in Physics vol. 39, pp. 218-223, Springer (1975)

43. Price, G.C., Williams, D.: Rolling with 'slipping'. Séminaire de Probabilités XVII (Paris), Lecture Notes in Mathematics 986, 194-297, Springer-Verlag, (1983)

44. van den Berg, M., Lewis, J.T.: Brownian motion on a hypersurface. Bull. Lond. Math. Soc. 17, 144-150 (1985)

45. Brillinger, D.R.: A Particle migrating randomly on a sphere. J. Theor. Probab. 10, 429-443 (1997)

46. Krishna, M.M.G., Samuel, J., Sinha, S.: Brownian motion on a sphere: distribution of solid angles. J. Phys. A: Math. Gen. 33, 5965-5971 (2000)

47. Debbasch, F., Espaze, D., Foulonneau, V.: Can diffusions propagate? J. Stat. Phys. 149, 37-49 (2012)

Publisher's Note Springer Nature remains neutral with regard to jurisdictional claims in published maps and institutional affiliations. 